\title{
THE ABUNDANCE OF ATOMIC ${ }^{1} \mathrm{H},{ }^{4}$ HE AND ${ }^{3}$ HE IN THE LOCAL INTERSTELLAR CLOUD FROM PICKUP ION OBSERVATIONS WITH SWICS ON ULYSSES
}

\author{
GEORGE GLOECKLER \\ Department of Physics and IPST, University of Maryland, College Park, MD 20742, USA, \\ and \\ Department of Atmospheric, Oceanic and Space Sciences, University of Michigan, \\ Ann Arbor, MI 48109, USA.
}

\begin{abstract}
Pickup ions measured deep inside the heliosphere open a new way to determine the absolute atomic density of a number of elements and isotopes in the local interstellar cloud (LIC). We derive the atomic abundance of hydrogen and the two isotopes of helium from the velocity and spatial distributions of interstellar pickup protons and ionized helium measured with the Solar Wind Ion Composition Spectrometer (SWICS) on the Ulysses spacecraft between $\sim 2$ and $\sim 5$ AU. The atomic hydrogen density near the termination shock derived from interstellar pickup ion measurements is $0.115 \pm 0.025 \mathrm{~cm}^{-3}$, and the atomic $\mathrm{H} / \mathrm{He}$ ratio from these observations is found to be $7.7 \pm 1.3$ in the outer heliosphere. Comparing this value with the standard universal $\mathrm{H} / \mathrm{He}$ ratio of $\sim 10$ we conclude that filtration of hydrogen is small and that the ionization fraction of hydrogen in the LIC is low.
\end{abstract}

\section{Introduction}

Pickup ions are created whenever slowly moving neutrals present in the solar system are ionized by charge exchange with the solar wind and solar UV radiation. In the heliosphere the major supply of neutrals is the local interstellar gas which enters the solar system at a relative speed of about 20 to $25 \mathrm{~km} / \mathrm{s}$ (Lallement et al., 1993; Witte et al., 1993) affected only by the force of gravity and by radiation pressure. These neutrals are ionized at distances sufficiently close to the sun and are embedded (picked up) in the expanding solar wind which sweeps them out of the solar system. Being charged, these newly born, interstellar pickup ions are now subjected not only to a variety of plasma processes that shape their distribution functions, but are also accelerated in the disturbed solar wind typically associated with transient shocks and corotating interaction regions (Gloeckler et al., 1994). At the heliospheric termination shock some fraction of these energetic pickup ions is further accelerated to many tens of $\mathrm{MeV}$ to become the so called 'anomalous cosmic rays'. Anomalous cosmic rays were discovered two decades ago (Garcia-Munoz et al., 1973; Hovestadt et al., 1973; McDonald et al., 1974) and recent experimental evidence indicates that they have low charge states (Cummings et al., 1993). Fisk et al. (1974) first proposed that anomalous cosmic rays are accelerated interstellar pickup ions. The average radial distance from the sun at which a large fraction of interstellar neutrals has been singly ionized is different for different interstellar atoms, depending on the ionization rates that in turn scale roughly as the first ionization potential of the atom. Helium atoms, whose ionization potential is high, penetrate as far in as $\sim 0.3 \mathrm{AU}$, whereas hydrogen atoms, and to a lesser degree oxygen, nitrogen and neon atoms are relatively rare at distances closer than several AU from the sun.

The first evidence for the presence of interstellar hydrogen and helium in the heliosphere was provided by measurements of resonantly scattered solar UV in the early 
1970s (Bertaux and Blamont, 1971; Thomas and Krassa, 1971). These and more recent measurements have been used to determine the density, temperature and relative velocity of interstellar hydrogen and helium with respect to the sun (Bertaux et al., 1985; Chassefière et al., 1986; Lallement et al., 1993; Quémerais et al., 1996). Interstellar neutral He has now been detected directly with a novel instrument flown on the Ulysses spacecraft (Witte et al., 1993).

In spite of the dynamical importance of pickup ions, particularly in the outer and distant heliosphere, attempts to detect them directly were unsuccessful until sensitive plasma composition instruments employing time-of-flight technology become available (Gloeckler et al., 1985; Gloeckler, 1990). Using an instrument of this type, interstellar pickup $\mathrm{He}^{+}$was detected at $1 \mathrm{AU}$ in 1985 (Möbius et al., 1985). The other pickup ions, namely $\mathrm{H}^{+},{ }^{4} \mathrm{He}^{++},{ }^{3} \mathrm{He}^{+}, \mathrm{N}^{+}, \mathrm{O}^{+}$and $\mathrm{Ne}^{+}$, were only recently discovered (Gloeckler et al., 1993; Geiss et al., 1994; Gloeckler and Geiss, 1996) using the Solar Wind Ion Composition Spectrometer (Gloeckler et al., 1992) on the Ulysses deep space probe.

Observations of interstellar pickup ions give us a new tool to probe the local interstellar medium, assess conditions in the vast regions beyond the heliospheric termination shock, and study a variety of heliospheric phenomena (see Table 1). While some of this work based on measurements of interstellar pickup ions has already been completed, much more is now in progress or remains to be done in the future. Table 1 summarizes some processes that may be studied and parameters that can be obtained from these new observations.

Table 1. Problems addressed with measurements of interstellar pickup ions

\begin{tabular}{|c|c|}
\hline Region & Processes or Parameters \\
\hline $\begin{array}{c}\text { Local } \\
\text { Interstellar } \\
\text { Cloud } \\
\text { Isotopic Ratios of } \mathrm{He}, \mathrm{Ne}\end{array}$ & $\begin{array}{l}\text { Relative Velocity and Temperature of } \mathrm{He}, \mathrm{O} \\
\text { Absolute Neutral Density of } \mathrm{H}, \mathrm{He} \\
\text { Fractional Ionization of } \mathrm{H}, \mathrm{He} \\
\text { Elemental Composition of Interstellar Gas }\end{array}$ \\
\hline $\begin{array}{l}\text { Heliospheric } \\
\text { Boundary Layer }\end{array}$ & $\begin{array}{l}\text { Filtration factor for } \mathrm{H}, \mathrm{O}, \mathrm{N} \\
\text { Pickup Ion Pressure }\end{array}$ \\
\hline Heliosphere & $\begin{array}{l}\text { Ionization Rates and Spatial Distribution } \\
\text { of Neutral Gas in the Heliosphere } \\
\text { Heliospheric and Termination Shock } \\
\text { Acceleration Processes } \\
\text { Transport and Plasma Processes }\end{array}$ \\
\hline
\end{tabular}

This paper focuses on observations of hydrogen and the two isotopes of helium. Their respective atomic abundance in the local interstellar cloud is derived from detailed observations of the spatial and velocity distributions of pickup protons and ionized helium (both $\mathrm{He}^{+}$and $\mathrm{He}^{++}$) at distances between $\sim 2$ and $\sim 5 \mathrm{AU}$ with the SWICS instrument on Ulysses.

To interpret observations of interstellar pickup ions (IPIs), it is useful to review briefly some of the basic characteristics of interstellar neutrals and IPIs in the heliosphere obtained from simple models developed in the early $1970 \mathrm{~s}$ (see, for example, Holzer, 1977, Thomas, 1978, and Vasyliunas and Siscoe, 1976) when the presence of 
interstellar hydrogen and helium throughout the heliosphere was established from UV observation.

\section{Spatial Distribution of Interstellar Neutrals in the Heliosphere}

The source material of the IPIs is the local interstellar cloud gas that makes its way deep into the heliosphere. The distribution in space of this gas within the heliosphere is reasonably well understood once the gas passes the heliospheric termination shock. The most appropriate model describing $n_{k}{ }^{*}(R, \Theta)=n_{k}(R, \Theta) / N_{k}$, the normalized spatial distribution of atoms of type $k$, in the heliosphere at radial distance $R$ from the sun and angle $\Theta$ with respect to the bulk flow direction, is the so-called 'hot model' (e.g. Thomas, 1978). The gas kinetic temperature, the appropriate ionization (loss) rate, $\beta_{k l o s s}$, for removing neutrals by ionization, and the ratio of radiation to gravitational forces, $\mu$, are parameters in this model, and $N_{k}$ is the number density of the gas just inside the termination shock. Of the three generally not well known parameters, uncertainties in the loss rate will have the greatest effect on the spatial distribution of neutrals. In fact, for atoms heavier than hydrogen, radiation pressure is insignificant compared to gravitation $(\mu=0)$ and the gas temperature plays only a minor role.

For hydrogen and some heavier atoms such as oxygen, the density inside the termination shock is believed to be less than the LIC density because of filtration (e.g. Baranov et al., 1993, 1995; Zank and Pauls, 1996) which prevents some of the interstellar gas from crossing the termination shock due to ionization. The filtration factor, $\left(N_{k} / N_{k \text { LIC }}\right)$ will depend on the proton density in the LIC and the relevant charge exchange ionization rate. Primarily because of uncertainties in our knowledge of the interstellar proton density, the amount of filtration is not well determined from models.

Filtration is unimportant for helium (Zank, personal communication). Since in addition helium is likely to be unaffected by possible changes in radiation pressure or uncertainties in its temperature, the only relevant model parameter for helium is its loss rate. Fortunately, the He loss rate is small $\left(<2 \cdot 10^{-7} \mathrm{~s}^{-1}\right)$ implying that at distances of $\sim 5 \mathrm{AU}$ from the sun the helium density will be as high as $90 \%$ of its interstellar value. For hydrogen, on the other hand, filtration effects may not be negligible, radiation pressure is comparable to gravitation, and the ratio of thermal to bulk speed is sufficiently large so that the gas temperature becomes also an important parameter. Because the hydrogen loss rate is large, its density is greatly reduced at distances smaller than $\sim 3-5 \mathrm{AU}$, and interstellar pickup hydrogen will be virtually undetectable at $1 \mathrm{AU}$.

\section{Model and Measured Velocity Distributions of Pickup Ions}

Interstellar pickup ions are created when interstellar atoms are ionized in the heliosphere. The number of IPIs produced is proportional to the ionization (production) rate, $\beta_{k \text { prod, }}$ at the time of ionization, and the local density, $n_{k}(R, \Theta)$, of neutral atoms. While it was often assumed that $\beta_{k \text { prod }}=\beta_{k \text { loss }}$, this is generally not the case as was pointed out by Gloeckler et al. (1993). This is because ionization rates vary with time and probably heliolatitude and the instantaneous production rate will not always equal the long-timeaveraged loss rate.

The distribution of IPIs in velocity space after pickup is rather complicated and at present not well modeled. However, the basic transformations that take place right after pickup are reasonably well understood. The newly ionized particles will gyrate about the 
local magnetic field to form a so-called ring distribution in velocity space as they are convected outward with the solar wind at the local solar wind speed, $V_{\text {sw }}$. In the solar wind frame this ring is at $V=\left|V_{\mathrm{sw}}\right|$ and pitchangle $\cos (\alpha)=\boldsymbol{V} \boldsymbol{B} /(V|\boldsymbol{B}|)$. After some time $\tau_{\mathrm{r}}$, the ring distribution should relax into a spherical shell distribution (with $V=\left|\boldsymbol{V}_{\mathrm{sw}}\right|$ ) due to pitchangle scattering. After some other time $\tau_{\mathrm{s}}$, the shell distributions would broaden due to energy diffusion. By assuming strong pitchangle scattering and little energy diffusion, that is $\tau_{\mathrm{r}}$ \& $R / V_{\mathrm{SW}}$ \& $\tau_{\mathrm{S}}$ Vasyliunas and Siscoe (1976) found that the distribution function in the solar wind frame is isotropic and can be expressed as

$$
\mathrm{f}_{\mathrm{vs}}(w<1, R, \Theta)=(3 / 8 \pi) V_{\mathrm{sw}}^{-4} N_{k} \beta_{k \text { prod }}\left(R_{\mathrm{o}}^{2} / R\right) w^{-3 / 2} n_{k} *\left(R w^{3 / 2}, \Theta\right) w<1
$$

where $w=v_{\text {ion }} / V_{\mathrm{sw}}$ and $R_{\mathrm{o}}=1.5 \cdot 10^{13} \mathrm{~cm}=1 \mathrm{AU}$. For $w>1 \mathrm{f}(w>1, R, \Theta)=0$. The strong pitchangle scattering implies adiabatic cooling which is incorporated in eq. (1).

The measured velocity distribution, $\mathrm{F}(W)$, of interstellar pickup ions is generally observed in only a limited volume of phase space in the spacecraft frame, and is usually integrated over all directions sampled by the instrument. Furthermore, time averaging over long periods (days to months) is often required to obtain sufficient statistical accuracy. $\mathbf{F}(W, R, \Theta)$ is derived from the measured count rate, $r_{m / q}(W, R, \Theta)$, of a given pickup ion (identified by its mass per charge $m / q$ and, in some cases mass) and the measured solar wind speed, with $W=V_{\text {ion }} / V_{\text {sw }}$, the speed of the ion in the spacecraft frame divided by the solar wind speed,

$$
\mathrm{F}(W, R, \Theta)_{m / q}=C_{\mathrm{inst}}\left\{r_{m / q}(W, R, \Theta) / \eta_{m / q}\left(V_{\mathrm{ion}}\right)\right\} V_{\mathrm{sw}}^{-4} W^{-4}
$$

$C_{\text {inst }}$ is the instrument factor (a product of the geometry factor, energy/charge bandwidth and conversion factors) and $\eta_{m / q}\left(V_{\text {ion }}\right)$ is the counting efficiency for that ion at its measured speed $V_{\text {ion. }}$ To compare the observed velocity distribution to model predictions (e.g. eq. 1), requires transformation to the spacecraft frame $\left(w=W-V_{\text {sw }} /\left|V_{\mathrm{sw}}\right|\right)$ and integration of the model distribution function over the instrument view angles, $\Omega_{\text {inst }}$

$$
\mathrm{F}(W, R, \Theta)_{m / q}=\int_{\Omega_{\mathrm{inst}}} d \Omega \mathbf{f}_{m / q}(w, \theta, \phi, R, \Theta) d \theta d \phi
$$

\section{Observations of Pickup Ion Velocity Distributions}

Initially pickup ion distributions of $\mathrm{He}^{+}$(Möbius et al., 1985) and $\mathrm{H}^{+}$as well as $\mathrm{He}^{+}$ (Gloeckler et al., 1993) were measured in a very limited range of ion speeds corresponding to $W>1.5$, and appeared to be adequately described by the isotropic velocity distribution function (eq. 1). A sharp drop in phase space density above $W=2$ was clearly observed. However, when it became possible to measure IPI distribution functions over a broader $W$ range (including speeds well below that of the solar wind, or $W<1$ ), distributions that were isotropic in the solar wind frame could no longer describe the measurements (Gloeckler et al., 1995). These new observations forced one to conclude that pitchangle scattering is weak (Gloeckler et al., 1995; Fisk et al., 1996; Möbius, 1996). Since in many instances IPI distributions are likely to be highly anisotropic, pickup ion fluxes, and estimates of interstellar abundances based on observations of distribution functions above $W \sim 1.5$ assuming isotropy, will lead to underestimates of the true atomic densities in the outer heliosphere. 
To illustrate these points, we show in Fig. $1 \mathrm{~F}(W)$ for pickup hydrogen measured with SWICS on Ulysses (see Gloeckler et al. (1992) for the description of the SWICS instrument, and Fig. 3 caption) in the south-polar coronal hole at $2.54 \mathrm{AU}$. The data were averaged over a 100-day period which was justified because solar wind conditions in these coronal hole regions are extremely steady. The solar wind proton speed was $780 \mathrm{~km} / \mathrm{s}$ and its density $0.4 \mathrm{~cm}^{-3}$. It is important to note that at these high latitudes the average magnetic field is almost radial and thus nearly aligned with the solar wind flow direction.

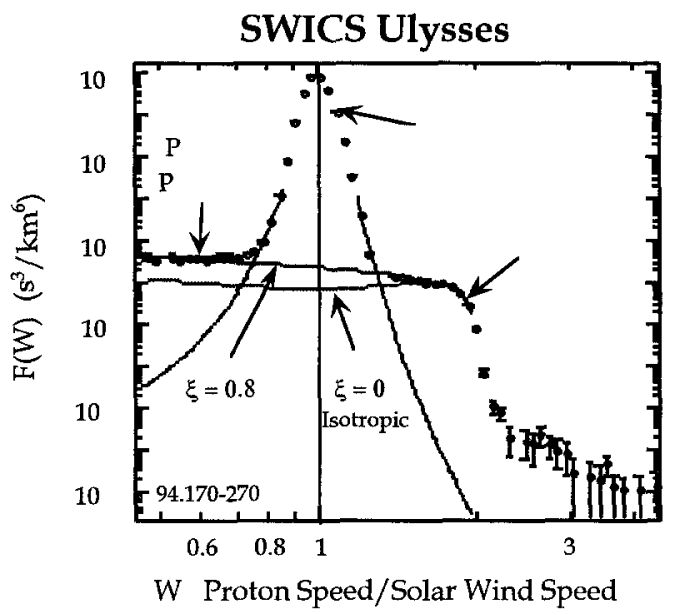

Fig. 1. Phase space density of protons measured with SWICS in the fast solar wind of the south-polar coronal hole. The velocity distribution of the hot pickup protons extends up to the cutoff at twice the solar wind speed and is well separated and distinctly different from the solar wind distribution. Beyond this sharp cutoff the density drops by three orders of magnitude. An isotropic (in the solar wind frame) distribution is ruled out by the observations. Counts accumulated below $W=1$ in the sun sector have not been included because of possible instrument background due to scattered solar wind protons.

Several characteristics of pickup ions can be established from this proton spectrum measured above the solar wind peak. First, the pickup proton spectrum extends all the way to the predicted cutoff at $W=2$. This implies that there is little if any thermalization of pickup ions, that is, the coupling between the solar wind plasma and pickup ions is so weak that little energy is exchanged as was first discussed by Feldman et al. (1974). Second, the drop-off of phase space density beyond the cutoff at $W=2$ (corresponding to $w=1$ in the solar wind frame) is very sharp, implying little if any energy diffusion. Thus, one can conclude that in the unperturbed solar wind, energy diffusion is negligible and $\tau_{\mathrm{s}}$ " $R / V_{\mathrm{sw}} \approx 10^{6} \mathrm{sec}$. The more than three orders of magnitude smaller phase space density above $W=2$ is probably a combination of a residual suprathermal tail and accidental low-level instrument background.

The anisotropy in the solar wind frame velocity distribution of the hydrogen IPI was revealed when its $\mathrm{F}(W)$ could be measured at speeds well below $V_{\mathrm{sw}}(W<1)$, that is for protons in the solar wind frame moving in the direction towards the sun. The curve labeled $\xi=0$ was obtained from eq. (3) using eq. (1) for $\mathrm{f}(w)$. Clearly, this curve falls a factor of three to four below the observations and is thus inconsistent with the measurements. To account for the observed excess of pickup protons below $W=1$ it is necessary to use anisotropic solar wind frame distributions. The curve labeled $\xi=0.8$ shows an example of an anisotropic distribution of the form

$$
\mathrm{f}(w<1, R, \Theta, \theta)=\mathrm{f}_{\mathrm{vs}}(w<1, R, \Theta)\{1+\xi \cos (\theta)\}
$$


where $\theta$ is the angle between the velocity, $w$, of the pickup ions in the solar wind frame and the inward radial direction. The anisotropy is the result of weak pitchangle scattering (Gloeckler et al., 1995; Möbius, 1996) and its magnitude, $\xi$, depends not only on the pitchangle scattering mean free path, $\lambda$, but also on the average time between the creation of the pickup ion and its detection. Thus, $\xi$ is likely to be larger for pickup $\mathrm{H}^{+}$than for $\mathrm{He}^{+}$because most protons observed at several AU have been produced relatively recently and thus had little time to isotropize, while most pickup He was ionized closer to the sun and hence had additional time to become more isotropic. Weak pitchangle scattering $(\lambda \approx 1 \mathrm{AU})$ implies that the coupling between pickup ions and the ambient solar wind is weak, and that the assumption $\tau_{\mathrm{r}} \ll R / V_{\mathrm{sW}} \approx 10^{6} \mathrm{sec}$ no longer holds.

With $\tau_{\mathrm{r}}$ comparable to the solar wind convection time of $\sim 10^{6} \mathrm{sec}$, the exact solution for $\mathrm{f}(\boldsymbol{w})$ becomes difficult and is currently not available (Fisk et al., 1996; Möbius, 1996). However, we now believed that in the case when the magnetic field is nearly radial, the ring distribution of newly created pickup ions in the inward $\left(\boldsymbol{w} \cdot V_{\mathrm{Sw}}<1\right)$ hemisphere of phase space initially decays with some time constant $\tau_{1}$. Then, with scattering suppressed near $90^{\circ}$ pitchangles, as seems to be indicated by pickup proton observations (Fisk et al., 1996), the phase space density in the outward $\left(w \cdot V_{\mathrm{SW}}>1\right)$ hemisphere becomes nearly isotropic but is reduced compared to that in the inward hemisphere by an amount that increases with $\lambda$. Adiabatic cooling still persists (Fisk et al., 1996). Lacking an exact solution we use eq. (4) which appears to be an adequate approximation to the true anisotropic solar wind frame distribution function, $\mathrm{f}(w)$ (see Fig. 1).

In the special case when the magnetic field is perpendicular to the solar wind flow direction (i.e. $\cos (\alpha) \approx 0$ ), as is often the case at radial distances beyond $\sim 5 \mathrm{AU}$ in the ecliptic plane, the phase space density in the two hemispheres should be about equal and no pronounced inward/outward anisotropy should be present.

\section{The Hydrogen to Helium Ratio Near the Termination Shock}

The spatial distribution of pickup ion fluxes, $\mathbf{J}_{k}$ (or densities) is approximately determined by the spatial distribution of interstellar neutrals $N_{k} n_{k}^{*}(\rho, \Theta)$ and the relevant production rates, $\beta_{k \text { prod }}$,

$$
\mathrm{J}_{k}(R, \Theta)=N_{k} \beta_{k \operatorname{prod}}\left(R_{\mathrm{O}} / R\right)^{2} \int_{0}^{R} n_{k}{ }^{*}(\rho, \Theta) d \rho .
$$

While $n_{k}^{*}(\rho, \Theta)$ is not directly measured as a function heliocentric radius $R$ it may be estimated from model predictions.

In Fig. 2 is shown the spatial gradient of pickup hydrogen measure with SWICS on Ulysses during the high-latitude pass over the south-polar regions of the sun. In order to minimize effects due to the large anisotropies in the distribution function, the $\mathrm{H}^{+} / \mathrm{He}^{+}$ flux ratio is plotted versus heliocentric distance. The dependence of this ratio on the ionization rates is shown in eqs. (6a) and (6b) below.

$$
\begin{aligned}
& \mathrm{J}_{\mathrm{H}}(R) / \mathrm{J}_{\mathrm{He}}(R)=\left\{\left(N_{\mathrm{H}^{3}} \beta_{\mathrm{H}^{+} \text {prod }}\right) /\left(N_{\mathrm{He}} \beta_{\mathrm{He}^{+} \text {prod }}\right)\right\} \bullet \mathbf{G}\left(R, \beta_{\mathrm{Hloss}}, \beta_{\mathrm{Heloss}}\right), \\
& \mathbf{G}\left(R, \beta_{\mathrm{Hloss}}, \beta_{\mathrm{Heloss}}\right)=\int_{0}^{R} n_{k}^{*}\left(\rho, \beta_{\mathrm{Hloss}}\right) d \rho / \int_{0}^{R} n_{k}^{*}\left(\rho, \beta_{\mathrm{Heloss}}\right) d \rho .
\end{aligned}
$$

During solar minimum conditions when these measurements were made, ionization loss rates were low (Rucinski et al., 1996) and thus a larger fraction of neutrals, espe- 
cially helium, made their way to heliocentric distances of less than 5 AU. For helium, the fraction was over 0.9 and therefore even large uncertainty in its loss rate would result in small error in $n_{k}{ }^{*}$. In computing $\mathbf{G}(R)$ we used the 'hot model' for protons with $\mu=1$, gas temperature of $9000 \mathrm{~K}$ and an average total production rate of $3.3 \cdot 10^{-7} \mathrm{~s}^{-1}$, the largest part of which was derived from the simultaneously measured solar wind proton flux. For helium we neglected radiation pressure $(\mu=0)$ and assumed $\beta_{\mathrm{He}^{+} \text {prod }}=0.7 \cdot 10^{-7} \mathrm{~s}^{-1}$. The other parameters are given in the caption of Fig. 2.

Changing the loss rate for helium by $\pm 30 \%$ had little effect on the shape of $\mathbf{G}(R)$ which is determined primarily by the hydrogen loss rate, $\beta_{\mathrm{Hloss}}$. The height of the gradient curve in Fig 2. is proportional to (a) the product of the $N_{\mathrm{H}} / N_{\mathrm{He}}$ density ratio at the termination shock, (b) the ratio of the respective production rates, and (c) the ratio of the hydrogen-helium anisotropy amplitudes. The best fit to the gradient measurements is for $\beta_{\text {Hloss }}=(5.5 \pm 0.7) \cdot 10^{-7} \mathrm{~s}^{-1}$ and $N_{\mathrm{H}} / N_{\mathrm{He}}=7.3_{-1.7}^{+2.2}$. A more accurate determination of the $N_{\mathrm{H}} / N_{\mathrm{He}}$ ratio comes from comparison of the observed velocity distributions of $\mathrm{H}^{+}$and $\mathrm{He}^{++}$(rather than $\mathrm{He}^{+}$) above W 1.4. Using this method Gloeckler et al. (1996) obtained $N_{\mathrm{H}} / N_{\mathrm{He}}=7.7 \pm 1.3$ from SWICS observations in the high-latitude coronal hole with $\beta_{\text {Hloss }}=5.5 \cdot 10^{-7} \mathrm{~s}^{-1}$. It is important to note that in their study the production rates of both $\mathrm{H}^{+}$and $\mathrm{He}^{++}$were determined and thus, the production rate of $\mathrm{He}^{+}$was also obtained. The values of the production rates of $\mathrm{H}^{+}, \mathrm{He}^{+}$and $\mathrm{He}^{++}$were found to be $(3.3 \pm 0.3) \cdot 10^{-7} \mathrm{~s}^{-1},(0.65 \pm 0.07) \cdot 10^{-7} \mathrm{~s}^{-1}$.and $(0.02 \pm 0.003) \cdot 10^{-7} \mathrm{~s}^{-1}$ respectively.

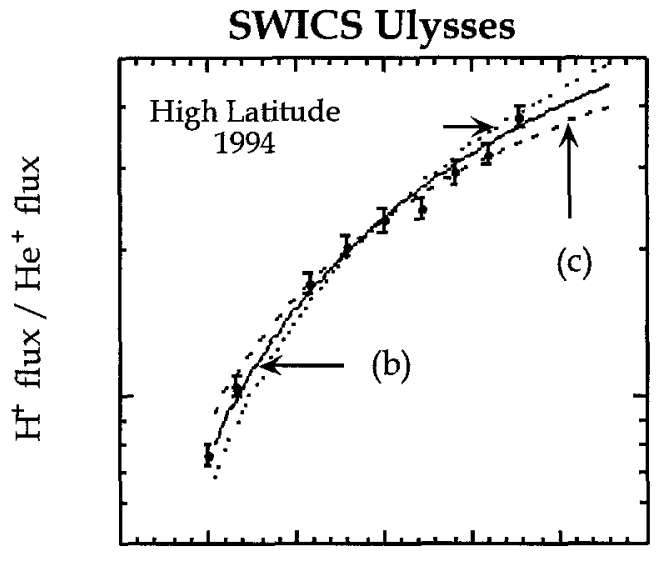

Fig. 2. Ratio of $\mathrm{H}^{+}$to $\mathrm{He}^{+}$pickup ion fluxes versus heliocentric distance. The flux ratio is derived from the observed phase space densities of $\mathrm{H}$ and $\mathrm{He}$ for $W$ between 1.75 and 2.0 and an assumed anisotropy ratio for $\mathrm{H}^{+} / \mathrm{He}^{+}$of $\sim 1.2$. Curves (a), (b) and (c) are model predictions of the flux ratio with $\beta_{\text {Hloss }}=$ $6.5 \cdot 10^{-7} \mathrm{~s}^{-1}, 5.5 \cdot 10^{-7} \mathrm{~s}^{-1}$, and $4.5 \cdot 10^{-7} \mathrm{~s}^{-1}$, and $N_{\mathrm{H}} / N_{\mathrm{He}}$ density ratios (near the termination shock) of $10.5,7.3$ and 5.0 respectively. Each data point represents a one-month average.

Heliocentric Distance (AU)

\section{The ${ }^{3} \mathrm{He} /{ }^{4} \mathrm{He}$ Abundance Ratio in the Local Interstellar Cloud}

The primordial abundances of the light elements and their isotopes provide essential information on processes of nucleosynthesis that occurred in the Big Bang (e.g. Schramm, 1993; Geiss, 1993). At present, the best estimates of the universal baryon/photon ratio, a fundamental constant of cosmology and the theory of matter, are extrapolations to primordial times of light-element abundances measured in the protosolar cloud and in the 
Milky Way. The first direct measurement of the ${ }^{3} \mathrm{He} / 4 \mathrm{He}$ abundance ratio in the LIC based on the isotopic analysis of interstellar pickup helium (Gloeckler and Geiss, 1996) gives a value of $(2.25 \pm 0.65) \cdot 10^{-4}$, and indicates little change in this ratio during the past $4.5 \cdot 10^{9}$ years. The observations are summarized in Fig. 3. Because all known physical processes that transform interstellar atoms into pickup ions found well inside the heliosphere are very likely to affect isotopes of the same element in similar fashion, the isotopic ratio of pickup ions measured close to the sun is the local interstellar cloud isotopic abundance.

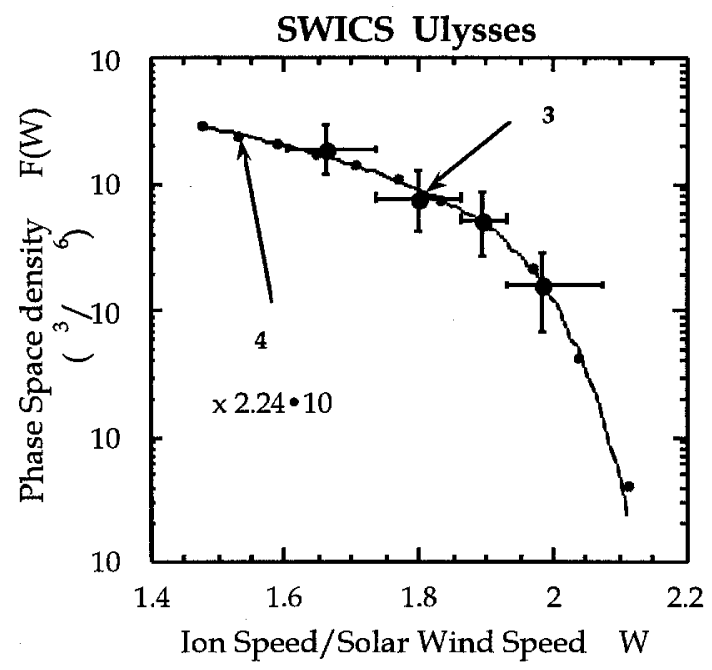

Fig. 3. (from Gloeckler and Geiss, (1996)). The measured distribution functions, $\mathrm{F}_{\mathrm{m}}(W)$, of interstellar pickup ${ }^{3} \mathrm{He}^{+}$and ${ }^{4} \mathrm{He}^{+}$(times $2.24 \cdot 10^{-4}$ ) versus the normalized ion speed, $W$. The phase space densities of ${ }^{3} \mathrm{He}^{+}(\mathrm{m}=3)$ and ${ }^{4} \mathrm{He}^{+}$ $(\mathrm{m}=4)$ were computed from their respective efficiencies $\eta_{\mathrm{m}}(W)$, and count rates $\mathrm{r}_{\mathrm{m}}(W)$, (with ${ }^{3} \mathrm{He}^{+}$corrected for a $10 \%$ background).

$\mathrm{F}_{\mathrm{m}}(W)=4541\left(\mathrm{r}_{\mathrm{m}} / \eta_{\mathrm{m}}\right)\left(438 / V_{\mathrm{sW}}\right)^{4} W^{-4}$. Despite relatively large statistical uncertainties in the four values of the ${ }^{3} \mathrm{He}^{+}$ phase space densities, the similarity of the two spectral shapes is striking. No ${ }^{3} \mathrm{He}^{+}$was observed above $W \AA 2$, consistent with the expected cutoff seen in the distribution functions of pickup ions. Below $W \AA 1.5$ the probability for triple coincidence becomes extremely small. SWICS measures the intensity of ions as a function of energy per charge (E), mass, and charge state, from 0.6 to $60 \mathrm{keV} \mathrm{e}-1$ in 64 logarithmically spaced steps with $\Delta \mathrm{E} / \mathrm{E} \AA 0.04$. Energy-per-charge analysis, followed by postacceleration of $23 \mathrm{kV}$, a time-of-flight determination and energy measurement with solid-state detectors is used to identify ions and sample their distribution functions once every 13 minutes. At solar-wind speeds $>750 \mathrm{~km} \mathrm{~s}^{-1}$ the energy of pickup ${ }^{3} \mathrm{He}^{+}$with speeds $>1.6 \cdot V_{\text {sw }}$ was typically above the $\sim 40 \mathrm{keV}$ threshold of the solid-state detectors, and the probability of obtaining triple coincidence analysis was thus high. Only triple coincidence data were used to obtain the velocity distribution functions of interstellar ${ }^{3} \mathrm{He}^{+}$and ${ }^{4} \mathrm{He}^{+}$shown here.

\section{Summary and Conclusions}

We have shown how measurements of interstellar pickup ions give new information on atomic abundances in the local interstellar cloud, as well as important insight on the interaction of low energy particles with the ambient solar wind. Far from the sun, pickup ions become dynamically important (Whang and Burlaga, 1996; Ness et al., 1996) and are likely to affect the structure of the outer heliosphere and the termination shock. There is no doubt that pickup ions are accelerated (Gloeckler et al., 1994) in the turbulent solar wind that often prevails in the near ecliptic region of the heliosphere as shown in Fig. 4. These pre-accelerated pickup ions are most likely the seed population of the anomalous cosmic rays, and detailed information on the velocity distribution of 
these pickup ions is essential for determining termination shock acceleration parameters, such as ion injection efficiencies.

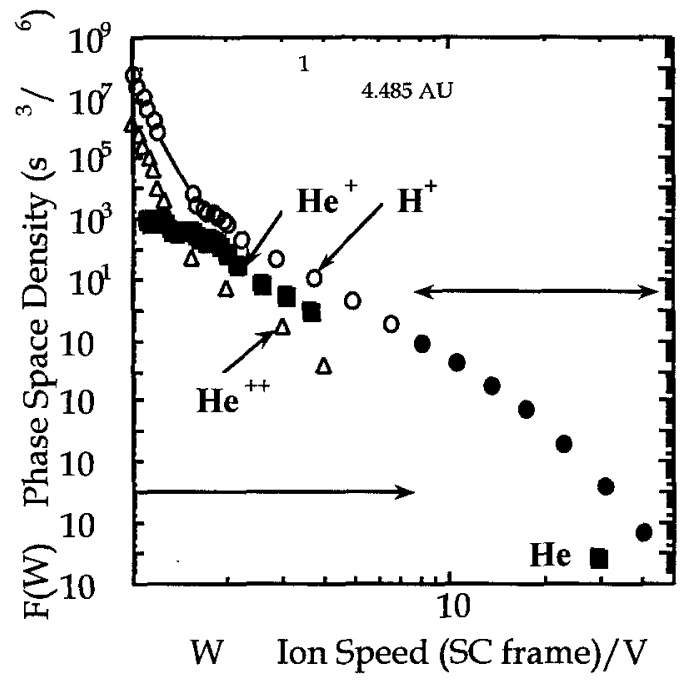

Fig. 4. (from Gloeckler et al., (1994)). Velocity distribution functions of $\mathrm{H}^{+}$(circles), $\mathrm{He}^{+}$(squares) and $\mathrm{He}^{++}$ (triangles) in the spacecraft frame. Data below $W \sim 8(0.6$ to $60 \mathrm{keV} / \mathrm{e})$ are obtained with SWICS, while those above $W$ $\sim 8$ are measured with the HI-SCALE instrument (Lanzerotti et al., 1992). Interstellar pickup $\mathrm{H}^{+}$appears as a bump on the spectrum below $W=2$. The accelerated solar wind $\mathrm{H}^{+}$and $\mathrm{He}^{++}(1.1<W$ $<1.5)$ have steep power law spectra with respective indices of $\sim-22$ and $\sim-30$. Accelerated pickup $\mathrm{H}^{+}$and $\mathrm{He}^{+}(W>2)$ have identical spectral shapes that are less steep than that of accelerated solar wind $\mathrm{He}^{++}$above $W \sim 1.5$ which is a power law of index $\sim-8$.

The light-element and isotope abundances in the LIC address fundamental questions of Big Bang nucleosynthesis and galactic chemical evolution. For example, the first measurements of the LIC ${ }^{3} \mathrm{He} / 4 \mathrm{He}$ abundance ratio are already placing constraints on how much ${ }^{3} \mathrm{He}$ is produced by low mass stars. The accuracy to which this ratio is determined is currently limited by low statistics. This will improve as additional data are acquired by a number instruments that can make these measurements. It should be possible to measure the ${ }^{22} \mathrm{Ne} / 20 \mathrm{Ne}$ abundance ratio from SWICS observations in the near future.

Our ability to determine accurate atomic abundances in the LIC is currently limited by systematic uncertainties due to poor knowledge of ionization rates, in particular the loss rates that govern the amount of depletion of the interstellar gas in the inner heliosphere where pickup ion measurements are made. These loss rates are time dependent, vary with solar activity (Rucinski et al., 1996) and may well be different in the highlatitude regions of the heliosphere, thus producing additional asymmetries in the spatial distribution of interstellar neutrals. Present uncertainties could be reduced with direct UV measurements (now done on SOHO with the Celias EUV He line monitor), or constrained by detailed observations of the spectral shapes and gradients of pickup ions, especially $\mathrm{H}^{+}$, as discussed in this paper. The last method is judged to be the more reliable for obtaining these unknown parameters, especially for hydrogen, because it is more direct and does not require a-priori knowledge of how to integrate and over what time intervals to average solar wind or UV fluxes generally measured at $1 \mathrm{AU}$ near the ecliptic plane.

We are in better shape when it comes to determining production rates. For $\mathrm{H}^{+}$the dominant part of the ionization is due to charge exchange with solar wind protons and this part is continuously measured. For $\mathrm{He}^{+}$the dominant part is due to photoionization which was not measured routinely until the launch of SOHO in late 1995. However, in their determination of the $\mathrm{H} / \mathrm{He}$ ratio, Gloeckler et al. (1996) were able to use SWICS 
observations of pickup $\mathrm{He}^{++}$produced almost entirely by double charge exchange with solar wind alpha particles. The solar wind alpha particle flux, just as the solar wind proton flux, was also measured simultaneously with the IPIs and thus the production rate of pickup $\mathrm{He}^{++}$was known. This removed the large uncertainty introduced when determining the atomic abundance of $\mathrm{He}$ from measurements of pickup $\mathrm{He}^{+}$(Möbius, 1996) whose production rate was not directly measured.

In Fig. 5 we present atomic abundances of hydrogen in the outer $(>\sim 50 \mathrm{AU})$ heliosphere and helium in the local interstellar cloud. The helium density, labeled 'Witte' is based on direct observations of atomic helium with the GAS instrument on Ulysses (Witte et al., 1993; Geiss and Witte, 1996) and falls in the middle of the SWICS range. The helium density labeled 'Möbius', is derived from $1 \mathrm{AU}$ observations of pickup $\mathrm{He}^{+}$ (Möbius et al., 1995) corrected for distribution function anisotropy effects (Möbius, 1996). The Möbius results are marginally consistent with the SWICS determination, but fall outside the limits of the Witte et al. (1993) range of values, possibly because of systematic uncertainties in the $\mathrm{He}^{+}$production rate. The range of hydrogen densities $\left(0.135 \pm 0.025 \mathrm{~cm}^{-3}\right)$ determined from Lyman $\alpha$ emission data (see Quémerais et al. (1996) for an excellent review of these observations) overlaps nicely with the SWICS hydrogen abundance. The interval of consistency of the three independent methods -pickup ions, direct measurements of neutral helium and UV emission -- is the small dark-shaded area.

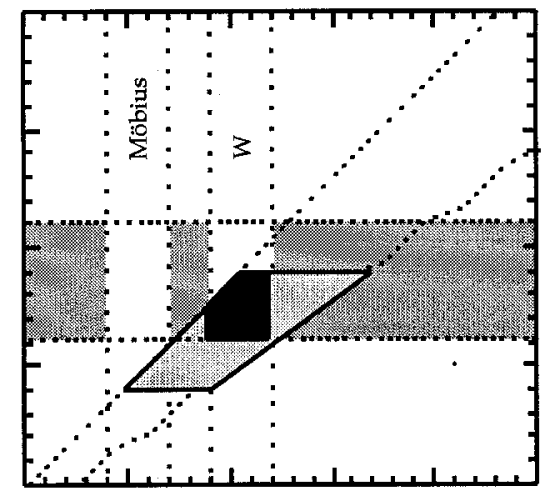

Atomic Helium

Fig. 5. Hydrogen versus helium density values derived from interstellar pickup ions, direct neutral helium measurements and UV observations. Ranges of four sets of observations are indicated by the various shaded regions. The small dark-shaded area is the region of consistency between abundances derived from pickup ion observations with SWICS (Gloeckler et al., 1996), UV observations (Quémerais et al., 1996) and atomic helium measurements (Witte et al., 1993).

Abundance in the $\mathrm{LIC}(\mathrm{cm} \quad)$

In Table 2 we list current best values for the abundance of the light elements and their isotopes (from Fig. 5) and the ionization rates for hydrogen and helium that were obtained from measurements of pickup ions with SWICS. The loss rates for hydrogen are determined to be different during the two phases of the solar cycle. However, in each case they fall well within the range given by Rucinski et al. (1996).

The hydrogen density near the termination shock $\left(\mathrm{N}_{\mathrm{H}}\right)$ obtained from a combination of pickup hydrogen and UV observations, is $0.125 \pm 0.015 \mathrm{~cm}^{-3}$, and the neutral helium density $\left(\mathrm{N}_{\mathrm{He}}=\mathrm{He}_{\mathrm{I}}\right)$ in the LIC, from direct detection of neutral helium, is $0.0155 \pm 0.0015 \mathrm{~cm}^{-3}$, giving a $\mathrm{N}_{\mathrm{H}} / \mathrm{He}_{\mathrm{I}}$ ratio of $8.1 \pm 1.3$. Comparing this with the standard universal $\mathrm{H} / \mathrm{He}$ abundance ratio of $\sim 10$ (Anders and Grevesse, 1988) implies little 
filtration of hydrogen and relatively low proton densities in the local interstellar cloud. A more precise determination of these parameters was made by Gloeckler et al. (1996). From their analysis of pickup ion measurements in combination with other observation and model calculations they find that the total hydrogen density in the LIC is $\sim 0.26 \mathrm{~cm}^{-}$ 3 , the filtration factor for hydrogen is $\sim 0.6$, and the fractional ionization of $\mathrm{H}$ and $\mathrm{He}$ is $\sim 0.15$ and $\sim 0.4$ respectively.

Table 2. Hydrogen and helium densities, abundance ratios and ionization rates in the outer heliosphere and the local interstellar cloud

\begin{tabular}{cccccc}
\hline \multirow{2}{*}{ Quantity } & Density $\left(\mathrm{cm}^{-3}\right)$, & \multicolumn{2}{c}{ Production Rate } & \multicolumn{2}{c}{ Loss Rate } \\
& or & \multicolumn{2}{c}{$\cdot\left(10^{-7} \mathrm{~s}^{-1}\right)^{*}$} & \multicolumn{2}{c}{$\cdot\left(10^{-7} \mathrm{~s}^{-1}\right)^{*}$} \\
& density ratio & 1991 & 1994 & 1991 & 1994 \\
\hline $\mathrm{N}_{\mathrm{H}}$ & $0.125 \pm 0.015^{\S}$ & $3.1 \pm 0.3^{\#}$ & $3.3 \pm 0.3$ & $8.5 \pm 1.0^{\#}$ & $5.5 \pm 0.5$ \\
$\mathrm{He}_{\mathrm{I}}$ & $0.0155 \pm 0.0015^{\dagger}$ & --- & $0.65 \pm 0.10^{\#}$ & -- & $0.6 \pm 0.1$ \\
$\mathrm{~N}_{\mathrm{H}} / \mathrm{He}_{\mathrm{I}}$ & $8.1 \pm 1.3 \S$ & & & & \\
$\left({ }^{3} \mathrm{He} /{ }^{4} \mathrm{He}\right)_{\text {LIC }}{ }^{\ddagger}$ & $(2.25 \pm 0.65) \cdot 10^{-4}$ & & & & \\
\hline
\end{tabular}

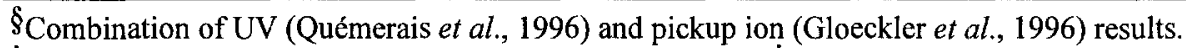

†From Witte et al. (1993) and Geiss and Witte (1996). \$From Gloeckler and Geiss (1996).

${ }^{*} \mu=1$ was used for hydrogen in 1991 and 1994.

\#From Gloeckler et al. (1996).

\section{Acknowledgments}

I am very grateful to the many individuals at the University of Maryland, the University of Bern, the Technical University of Braunschweig, the Max-Planck-Institut für Aeronomie, and the Goddard Space Flight Center who have contributed to the success of the SWICS experiment during the many years of its development. Of particular benefit to me have been the many insightful discussions with Johannes Geiss and Len Fisk. I thank Christine Gloeckler for her help with data reduction and preparation of the manuscript, and Daniel Rucinski for making his hot model subroutine available to me. This work was supported by NASA/JPL contract 955460 .

\section{References}

Anders, E. and Grevesse, N.: 1988, Geochim. Cosmochim. Acta 53, 1197.

Baranov, V. B. and Malama, Yu. G.: 1993, J. Geophys. Res. 98, 15157-15163.

Baranov, V. B. and Malama, Y. G.: 1995, J. Geophys. Res. 100, 755-761.

Bertaux, J.-L. and Blamont, J. E.: 1971, Astron. Astrophys. 11, 200.

Bertaux, J.-L., Lallement, R., Kurt, V. G., and Mironova, E. N.: 1985, Astron. Astrophys. 150, 1.

Chassefière, E., Bertaux, J.-L., Lallement, R., and Kurt, V. G.: 1986, Astron. Astrophys. 160, 299.

Cummings, J. R., Cummings, A. C., Mewaldt, R. A., Selesnick, R. S., Stone, E. C., and von Rosenvinge, T. T.: 1993, Geophys. Res, Lett. 20, 2003.

Feldman, W. C., Asbridge, J. R., Bame, S. J., and Kearney, P. D.: 1974, J. Geophys. Res. 79, 1808.

Fisk, L. A., Koslovsky, B., and Ramaty, R.: 1974, Astrophys, J. Lett. 190, L35.

Fisk, L. A., Schwadron, N. A., and Gloeckler, G.: 1996, Geophys. Res. Lett., (in press). 
Garcia-Munoz, M., Mason, G. M., and Simpson, J. A.: 1973, Astrophys. J. Lett. 182, L81.

Geiss, J.: 1993, in Origin and Evolution of the Elements (eds. Prantzos, N, Vangioni-Flam, E. \& Cassé, M.) 89-106 (Cambridge University Press).

Geiss, J., Gloeckler, G., Mall, U., von Steiger, R., Galvin, A. B., and Ogilvie, K. W.: 1994, Astr. Astrophys. 282, 924-933.

Geiss, J. and Witte, M.: 1996, Space Sci. Rev., this issue.

Gloeckler, G., et al.: 1985, IEEE Trans. on Geosci. and Remote Sensing, GE-23, 3, 234-240.

Gloeckler, G.: 1990, Rev. Sci. Instrum. 61, 23613-23620

Gloeckler, G., et al.: 1992, Astr. Astrophys. Suppl. Ser. 92, 267-289.

Gloeckler, G., et al:: 1993, Science 261, 70.

Gloeckler, G., et al.: 1994, J. Geophys. Res. 99, 17,637-17,643.

Gloeckler, G., Schwadron, N. A., Fisk, L. A., and Geiss, J.: 1995, Geophys. Res. Lett. 22, 26652668.

Gloeckler, G. and Geiss, J.: 1996, Nature 381, 210.

Gloeckler, G., Geiss, J, and Fisk, L. A.: 1996, submitted to Nature.

Holzer, T. E.: 1977, Rev. of Geophys. and Space Phys. 15, 467.

Hovestadt, D., Vollmer, O., Gloeckler, G., and Fan, C. Y.: 1973, Phys. Rev. Lett.. J. 31, 650.

Lallement, R., Bertaux, J.-L., and Clarke, J. T.: 1993, Science 260, 1095.

Lanzerotti. L. J., et al.: 1992, Astron. Astrophys. Suppl. Ser. 92, 349-365.

McDonald, F. B., et al.: 1974, Astrophys. J. Lett. 185, L105.

Möbius, E., Rucinski, D., Hovestadt, D., and Klecker, B.: 1995, Astron. Astrophys. 304, 505.

Möbius, E. et al.: 1985, Nature 318, 426.

Möbius, E.: 1996, Space Sci. Rev., this issue.

Ness, N. F., et al.: 1996, Space Sci. Rev., this issue.

Quémerais, E., Bertaux, J._L., Sandel, B. R., and Lallement, R.: 1996, Astron. Astrophys. 290, 961.

Rucinski, D., Cummings, A. C., Gloeckler, G., Lazarus, A. J., Möbius, E. and Witte, M.: 1996, Space Sci. Rev., this issue.

Schramm, D. N.: 1993 in Origin and Evolution of the Elements (eds. Prantzos, N., Vangioni-Flam, E. \& Cassé, M.) 112-131 (Cambridge University Press).

Thomas, G. E. and Krassa, R. F.: 1971, Astron. Astrophys. 11, 218.

Thomas, G. E.: 1978, Ann. Rev. Earth Planet. Sci. 6, 173.

Vasyliunas, V. M. and. Siscoe, G. L.: 1976, J. Geophys. Res. 81, 1247.

Whang, Y. C. and Burlaga, L. F.: 1996, Space Sci. Rev., this issue.

Witte, M., Rosenbauer, H., Banaszkiewicz, M. and Fahr, H. J.: 1993, Adv. Space Res. 13, (6)121.

Zank, G. P. and Pauls, H. L: 1996, Space Sci. Rev., this issue. 